\title{
Refined Composite Multivariate Multiscale Fractional Fuzzy Entropy: Measuring the Dynamical Complexity of Multichannel Financial Data
}

\author{
Huiqin Chu, ${ }^{1}$ Zhiyong $W u,{ }^{1}$ and Wei Zhang $\mathbb{D}^{2}$ \\ ${ }^{1}$ School of Mathematics-Physics and Finance, Anhui Polytechnic University, Wuhu, Anhui 241000, China \\ ${ }^{2}$ Key Laboratory of Advanced Perception and Intelligent Control of High-End Equipment, \\ Ministry of Education and School of Mathematics-Physics and Finance, Anhui Polytechnic University, Wuhu, \\ Anhui 241000, China \\ Correspondence should be addressed to Wei Zhang; slzw@ahpu.edu.cn
}

Received 28 July 2021; Accepted 31 August 2021; Published 10 September 2021

Academic Editor: Huihua Chen

Copyright (C) 2021 Huiqin Chu et al. This is an open access article distributed under the Creative Commons Attribution License, which permits unrestricted use, distribution, and reproduction in any medium, provided the original work is properly cited.

\begin{abstract}
Refined composite multivariate multiscale fractional fuzzy entropy (RCmvMFFE), which aims to sensitively discriminate different short noisy multichannel financial data, is proposed as a new measure to quantify the complexity dynamics of multichannel time series in this work. To better comprehend the RCmvMFFE measure, the dynamical complexity analyses of multichannel synthetic dataset are comparatively studied with multivariate multiscale fuzzy entropy (mvMFE), refined composite multivariate multiscale fuzzy entropy (RCmvMFE), and refined composite multivariate multiscale fractional fuzzy entropy (RCmvMFFE). Then, these measures are firstly employed to explore actual multichannel financial index series to the best of our knowledge. The empirical analyses report that RCmvMFFE measure is able to deeply and sensitively dig up the market information hidden in the multichannel financial data and can better discriminate markets in different area compared to the traditional measures to some extent.
\end{abstract}

\section{Introduction}

Financial market is a typical complex dynamical system, and financial time series is believed to possess strong nonlinearity $[1,2]$, nonstationarity $[3,4]$, multifractality $[5,6]$, longrange correlation, and structural dynamics [7-9]. Digging up the statistical characteristics hidden in financial variables (price, volume, etc.) is going through a vital important time in theoretical and applied research fields, especially in risk management and modeling market dynamics [10]. The complexity measure is such a statistical value related to the unpredictability or structural dynamics of a time series. The larger complexity measure of a signal means it possesses more randomness in small time scale and more structural dynamics in large time scale. As a matter of fact, $1 / f$ noise (holding long-range correlation) is more regular and possesses more structural information than white Gaussian noise (WGN) [8]. In the past thirty years, complexity analysis has gone a rapid improvement, and a lot of complexity measures and improved versions have sprung up. Among them, the most prominent one is entropy measure, for its simplicity and convenience implementation in modern computers. Entropy methods, such as approximate entropy (AppEn) [11, 12], sample entropy (SampEn) $[12,13]$, permutation entropy (PermEn) [14-16], and fuzzy entropy (FuzzyEn) $[13,17,18]$, have been successively introduced to explore the dynamical complexity hidden in financial and physiological data, which are commonly described as short and noisy series [19-21]. Among them, AppEn measures regularity or randomness of a series through constructing difference of mean logarithmic function of the probability of vector pairs within tolerance $r$ of template vectors with two adjacent integer embedding dimensions [11]. SampEn, which is a revised method of 
AppEn, overcomes the dependency on parameters by excluding self-matching [12]. Moreover, FuzzyEn is also a revised version of SampEn by introducing continuous differentiable exponential fuzzy membership function to overcome the sensitivity to parameters, tolerance $r$ in particular [13]. While structural property is universal in nature $[8,22]$, some counterintuitive results are reported in applications with the above single-scale entropy measures for inherent natural multiple spatial and temporal scales in complex dynamics system, so multiscale methods are proposed in response to the proper time and conditions. While it is found that traditional methods cannot distinguish different time series very well, fractional fuzzy entropy (FFE) [18] is proposed by combining entropy measure with fractional calculus [23-25], and it can dig up fractional order information hidden in a nonlinear complex system sensitively and can obtain more exact and deep understanding. Composite multiscale entropy family has been put forward by combining the conventional entropy methods with refined and composite technique to estimate dynamical complexity of short-term financial data, and they show significant superiority in reliability and stability, such as refined composite multiscale permutation entropy (RCMPE) and refined composite multiscale fuzzy entropy (RCMFE) [19, 26]. Moreover, the real world is essentially multivariate, financial data in particular. Multivariate entropy is proposed to analyze complexity of multichannel data and has been utilized to detect multichannel physiological and environmental series [27], and it is firstly used to discuss the dynamical complexity of multichannel financial time series compared with RCmvMFFE to the best of our knowledge.

In this work, inspired by the influential studies $[17,20,27]$, combining multivariate entropy with fractional calculus, multiscale, and composite technique, a developed complexity version, refined composite multivariate multiscale fractional fuzzy entropy (RCmvMFFE), is proposed to explore and discriminate the complexity dynamics of multichannel time series. Moreover, through the analyses of the multichannel synthetic dataset and financial data, the experimental results validate that the proposed RCmvMFFE displays a better performance compared to traditional benchmark complexity measures to some extent.

The remainder of this paper is organized as follows. Section 2 reviews the FuzzyEn, FFE, RCMFE, RCmvMFE, and RCmvMFFE methods briefly. Section 3 evaluates the effectiveness of mvMFE, RCmvMFE, and RCmvMFFE with multichannel synthetic dataset. Section 4 presents the complexity analysis results of multichannel financial time series in different regions, followed by conclusions in Section 5 .

\section{Methodologies}

2.1. Fractional Fuzzy Entropy. Fuzzy entropy (FuzzyEn), which is a revised complexity measure of SampEn based on vectors shapes and fuzzy membership function family, shows significant advantages in reliability and stability to noise and freedom to parameters selection and gets widespread applications in physiological and financial fields. The highlight of FuzzyEn is that continuous differentiable exponential function family in place of rigid Heaviside function is used to evaluate similarity degree between vectors to avoid the sensitiveness to parameter selection.

The FuzzyEn algorithm can be briefly reviewed as follows; for more details, see $[13,17]$. Given a time series with length $T, x=\left\{x_{i}, i=1,2, \ldots, T\right\}$, firstly an $m$-dimensional vector series, $\left\{X_{i}^{m}, 1 \leq i \leq T-m+1\right\}$, is reconstructed by the well-known phase space reconstruction theory [28], and each subtracts its mean value to eliminate the influence of tendency as follows:

$$
X_{i}^{m}=\left(x_{i}, x_{i+1}, \ldots, x_{i+m-1}\right)-\bar{x}(i),
$$

where $m$ is predefined embedding dimension which controls the amount of information each vector conveys and $\bar{x}(i)$ is the arithmetic mean of the vector $\left(x_{i}, x_{i+1}, \ldots, x_{i+m-1}\right)$ for baseline removal defined as follows:

$$
\bar{x}(i)=\frac{1}{m} \sum_{j=0}^{m-1} x_{i+j} .
$$

Every point in phase space represents an instantaneous state of a system considered. Then, for vector series $\left\{X_{i}^{m}\right\}$ reconstructed above, the similarity degree $D_{i j}^{m}$ of an arbitrary $X_{i}^{m}$ to another $X_{j}^{m}$ is calculated by a fuzzy membership map family as follows:

$$
D_{i j}^{m}=e^{-\left(d_{i j}^{m} / r\right)^{n}}
$$

where the predefined parameter $n$ represents the gradient of boundary and controls the weight of vector similarity, $r$ the width of the fuzzy function, and $d_{i j}^{m}$ the infinite norm of the $m$-dimension difference vector $X_{i}^{m}-X_{j}^{m}$ in the work.

Average of all the similarity degrees $D_{i j}^{m}$ and ensemble similarity degree $C^{m}(r)$ of any vector pairs in the mean sense can be obtained as follows:

$$
C^{m}(r)=\frac{1}{T-m} \sum_{i=1}^{T-m}\left(\frac{1}{T-m-1} \sum_{j=1, j \neq i}^{T-m} D_{i j}^{m}\right) .
$$

Analogously, for $m+1$ dimension case, with $\left\{X_{i}^{m+1}, 1 \leq i \leq T-m\right\}$ reconstructed when next point is added, the ensemble similarity degree $C^{m+1}(r)$ can be also obtained as follows:

$$
C^{m+1}(r)=\frac{1}{T-m} \sum_{i=1}^{T-m}\left(\frac{1}{T-m-1} \sum_{j=1, j \neq i}^{T-m} D_{i j}^{m+1}\right) .
$$

Finally, for the given $x$ with finite length, the FuzzyEn is estimated as follows:

$$
\operatorname{FuzzyEn}(x, m, n, r)=\ln C^{m}(r)-\ln C^{m+1}(r)=-\ln \frac{C^{m+1}(r)}{C^{m}(r)}
$$

Then, fractional fuzzy entropy, which is a developed version of FuzzyEn measure, is introduced to sensitively dig up fractional order dynamics of a complex system by 
combining FuzzyEn with fractional calculus $[23,24]$. The fractional fuzzy entropy (FFE) of the given series $x$ is estimated as follows [18]:

$$
\operatorname{FFE}(x, m, n, r, \alpha)=-\frac{\ln C^{m+1}(r)-\ln C^{m}(r)+\psi(1)-\psi(1-\alpha)}{\Gamma(\alpha)}\left[\frac{C^{m+1}(r)}{C^{m}(r)}\right]^{-\alpha},
$$

where $\alpha \in[-1,1]$ represents the order of fractional derivative, $\Gamma(\cdot)$ the gamma function, and $\psi(\cdot)$ the digamma function with the form $\psi(x)=\Gamma^{\prime}(x) / \Gamma(x)$, respectively. Furthermore, FFE degenerates into conventional FuzzyEn as $\alpha \longrightarrow 0$.

Conventionally, the hyperparameters $m$ and $n$ are preset to be two small positive integers for avoiding the loss of information, $m$ is suggested to be between 3 and 7 [12], $n$ is fixed to be 2 in the work, and tolerance $r$ is set to be $0.15 \times \mathrm{SD}$, where $\mathrm{SD}$ presents the standard deviation of the original series.

\subsection{Refined Composite Multiscale Fuzzy Entropy. With the} extensive applications of entropy measures in more and more fields, some counterintuitive results are reported in single-scale entropy analysis for not considering the natural complex spatial and temporal scales inherent in complex systems considered, especially when pathologic signals are compared to healthy signals [8]. Multiscale entropy is proposed by combining the concept of coarse graining with entropy analysis, to measure the multiscale structural information inherent in dataset, for example, multiscale sample entropy (MSE) and multiscale fuzzy entropy (MFE) $[8,14]$. Refined composite multiscale fuzzy entropy (RCMFE) is proposed by calculating FuzzyEn values with averaging similarity degree of multiple embedded vector series with different starting points originated by the Takens embedding theorem to overcome the instability of the above MFE measure [19]. The algorithm of RCMFE for a given $x=\left\{x_{i}, i=1,2, \ldots, T\right\}$ mainly consists of the following two steps:

(1) Coarse graining for multiscale evaluation of fuzzy entropy: a "coarse graining" process with scale factor $\beta$ is firstly utilized for the original time series $x$ to get $\beta$ coarse-grained sequences, and the corresponding $k$-th sequence $y_{k}^{(\beta)}=\left\{y_{1, k}^{(\beta)}, y_{2, k}^{(\beta)}, \ldots, y_{[T / \beta]-1, k}^{(\beta)}\right\}$ is defined as follows:

$y_{i, k}^{(\beta)}=\frac{1}{\beta} \sum_{j=(i-1) \beta+k}^{i \beta+k-1} x_{j}, \quad 1 \leq i \leq\left\lfloor\frac{T}{\beta}\right\rfloor-1,1 \leq k \leq \beta$.

where \lfloor\rfloor represents rounding toward negative infinity.

(2) Calculating refined composite multiscale fuzzy entropy of sequences considered: for a fixed scale factor $\beta$, the two corresponding values $C_{k, \beta}^{m}(r)$ and $C_{k, \beta}^{m+1}(r)$ are, respectively, calculated for sequence $y_{k}^{(\beta)}=\left\{y_{i, k}^{(\beta)}, 1 \leq i \leq\lfloor T / \beta\rfloor-1\right\} \quad$ with embedding dimension $m$ and $m+1$ based on the above MFE. Then, the mean of $C_{k, \beta}^{m}(r)$ and $C_{k, \beta}^{m+1}(r)$ for all indexes $k$ denoted as $\bar{C}_{\beta}^{m}(r)$ and $\bar{C}_{\beta}^{m+1}(r)$ are computed, respectively; i.e., $\bar{C}_{\beta}^{m}(r)=1 / \beta \sum_{k=1}^{\beta} C_{k, \beta}^{m}(r)$, and $\bar{C}_{\beta}^{m+1}(r)=1 / \beta \sum_{k+1}^{\beta} C_{k, \beta}^{m+1}(r)$. Finally, RCMFE can be estimated as follows:

$$
\operatorname{RCMFE}(x, \beta, m, n, r)=-\ln \frac{\bar{C}_{\beta}^{m+1}(r)}{\bar{C}_{\beta}^{m}(r)} .
$$

In particular, the RCMFE degenerates into traditional MFE measure when $\beta=1$.

\subsection{Refined Composite Multivariate Multiscale Fractional} Fuzzy Entropy. Recent rapid developments in multimodal sensing and computer science have enhanced the necessity for more insight into the dynamical system considered, financial system in particular. Multivariate entropy (mvEn) generalizes univariate entropy to analyze complexity dynamics of multichannel data, and it has been widely used to analyze real-world multichannel physiological and environmental data. The highlight of mvEn is considering all the composite delay vectors, which are reconstructed by multivariate embedding theory [29], in the $p$ subspaces and then comparing them within and across the $p$ channels.

The multivariate multiscale fuzzy entropy (mvMFE) is improved by combining coarse graining and multivariate fuzzy entropy. The mvMFE algorithm is briefly described as follows for a given multivariate data $\left\{x_{k, i}\right\}_{i=1}^{T}, k=1,2, \ldots, p$, where $T$ represents the number of samples in each channel and $p$ the number of variates (i.e., channels).

(i) Define coarse graining series with a fixed temporal scale. Given a scale $\beta$, the elements of the multichannel coarse-grained series are defined as follows:

$$
z_{k, j}^{(\beta)}=\frac{1}{\beta} \sum_{i=(j-1) \beta+1}^{j \beta} x_{k, i}, \quad 1 \leq j \leq J=\left\lfloor\frac{T}{\beta}\right\rfloor, 1 \leq k \leq p .
$$

(ii) For a $p$-variate time series $\left\{z_{k, j}^{(\beta)}\right\}_{j=1}^{J}$, form $(J-N)$ composite delay vectors $Z_{m}(i) \in R^{m}$ by the multivariate embedded reconstruction theory as follows: 


$$
Z_{m}(i)=\left[z_{1, i}, z_{1, i+\tau_{1}}, \ldots, z_{1, i+\left(m_{1}-1\right) \tau_{1}}, z_{2, i}, z_{2, i+\tau_{2}}, \ldots, z_{2, i+\left(m_{2}-1\right) \tau_{2}}, \ldots, z_{p, i}, z_{p, i+\tau_{p}}, \ldots, z_{p, i+\left(m_{p}-1\right) \tau_{p}}\right]
$$

where $N=\max \{M\} \times \max \{\tau\}, \quad M=\left[m_{1}, m_{2}, \ldots\right.$, $\left.m_{p}\right]$ is corresponding embedding vector, $m=\sum_{k=1}^{p} m_{k}$ embedding dimension, and $\tau=\left[\tau_{1}, \tau_{2}\right.$ $\left., \ldots, \tau_{p}\right]$ time delay vector.

(iii) Calculate the distance $d_{i j}^{m}$ for any composite delay vector pairs $Z_{m}(i)$ and $Z_{m}(j)$ as the maximum norm of the difference vector $Z_{m}(i)-Z_{m}(j)$. For any given $Z_{m}(i)$ and a threshold $r$, the similarity degree $D_{i j}^{m}$ of $Z_{m}(i)$ to $Z_{m}(j)$ is defined as $D_{i j}^{m}=e^{-\left(d_{i j}^{m} / r\right)^{n}}$; then, define a global quantity $B^{m}(r)$ as the average membership degree as follows:

$B^{m}(r)=\frac{1}{J-N} \sum_{i=1}^{J-N}\left(\frac{1}{J-N-1} \sum_{j=1, j \neq i}^{J-N} D_{i j}^{m}\right)$.

(iv) Extend the embedding dimension in (11) from $m$ to $(m+1)$. This can be performed in $p$ different patterns, for example, from $M=\left[m_{1}, m_{2}, \ldots\right.$, $\left.m_{k}, \ldots, m_{p}\right]$ to $\left[m_{1}, m_{2}, \ldots, m_{k}+1, \ldots, m_{p}\right]$ for any $k=1, \ldots, p$.

(v) Calculate similarly $B^{m+1}(r)$ which represents the average of all similarity degrees $D_{i j}^{m+1}(r)$ in $(m+1)$-dimensional phase space. Finally, mvMFE is estimated as follows:

$\operatorname{mvMFE}(x, \tau, n, r)=\ln B^{m}(r)-\ln B^{m+1}(r)=-\ln \frac{B^{m+1}(r)}{B^{m}(r)}$.
As mentioned above, according to the proposed refined composite technique, the refined composite multivariate multiscale entropy-based approach firstly generates $\beta$ coarse-grained multivariate time series $Z_{u}^{(\beta)}=\left\{z_{k, j}^{(\beta, u)}\right\}$, $1 \leq u \leq \beta$, as follows:

$$
z_{k, j}^{(\beta, u)}=\frac{1}{\beta} \sum_{i=(j-1) \beta+u}^{j \beta+u-1} x_{k, i}, \quad 1 \leq j \leq J, 1 \leq k \leq p .
$$

For each scale $\beta, \beta$ different multivariate signals $Z_{u}^{(\beta)}$ with different starting points are obtained. For each multivariate sequence $Z_{u}^{(\beta)}$, membership degrees $B_{\beta, u}^{m}(u=1, \ldots, \beta)$ and $B_{\beta, u}^{m+1}(u=1, \ldots, \beta)$ are separately calculated. Next, the average values $\bar{B}_{\beta, u}^{m}$ and $\bar{B}_{\beta, u}^{m+1}$ on scale factor $1 \leq u \leq \beta$ are calculated. Then, the RCmvMFE is estimated as follows:

$$
\operatorname{RCmvMFE}(y, \beta, M, n, r)=-\ln \frac{\bar{B}_{\beta, u}^{m+1}}{\bar{B}_{\beta, u}^{m}}
$$

Finally, the corresponding RCmvMFFE value is calculated as follows:

$$
\operatorname{RCmvMFFE}(y, \beta, M, n, \alpha, r)=-\frac{\ln \bar{B}_{\beta, u}^{m+1}-\ln \bar{B}_{\beta, u}^{m}+\psi(1)-\psi(1-\alpha)}{\Gamma(\alpha+1)}\left[\frac{\bar{B}_{\beta, u}^{m+1}}{\bar{B}_{\beta, u}^{m}}\right]^{-\alpha}
$$

where $\alpha \in[-1,1]$ and the RCmvMFFE degenerates into traditional RCmvMFE measure as $\alpha \longrightarrow 0$.

\section{Validation of RCmvMFFE for Multichannel Synthetic Dataset}

In this section, we explore the complexity dynamics of threechannel synthetic dataset whose channels consist of Gaussian white noise (WGN) and $1 / f$ noise. We first apply mvMFE, RCmvMFE, and RCmvMFFE to analyze this dataset, which are commonly utilized to assess the performance of entropy-based methods. All three channels are independent, and the number of sample points of each channel is 5500 . We choose four datasets with different constitutions of WGN and $1 / f$ noise: all three channels contain $1 / f$ noise (group 1); two channels contain $1 / f$ noise and one WGN (group 2); one channel contains $1 / f$ noise and others WGN (group 3); and all three channels contain WGN (group 4). The corresponding mvMFE, RCmvMFE, and RCmvMFFE results are shown in Tables 1-6 and Figures 1 and 2.

The mean mvMFE and RCmvMFE values of the threechannel synthetic dataset (with 20 independent simulations) with different time scales $\beta=1,5,10,15,20,25,30,35,40$ are listed in Tables 1 and 2, and the corresponding standard deviation is presented in Tables 3 and 4 . For each synthetic dataset, the two kinds of entropy values decrease as the scales increase, respectively. The standard deviation values gradually increase as the scales increase (except for group 4), which could be a shorter coarse grain series for a larger time scale $\beta$. Moreover, for a given scale $\beta \geq 5$, entropy measures for group 1, group 2, group 3, group 4 are from big to small, which indicates that multichannel series with more $1 / f$ noise 
TABLE 1: mvMFE of three-channel synthetic dataset with different $\beta$.

\begin{tabular}{lccccccrrr}
\hline$\beta$ & 1 & 5 & 10 & 15 & 20 & 25 & 30 & 35 & 40 \\
\hline Group 1 & 0.3895 & 0.3247 & 0.3040 & 0.2906 & 0.2784 & 0.2703 & 0.2631 & 0.2544 & 0.2508 \\
Group 2 & 0.4251 & 0.2822 & 0.2549 & 0.2440 & 0.2373 & 0.2287 & 0.2243 & 0.2215 & 0.2149 \\
Group 3 & 0.4403 & 0.2066 & 0.1699 & 0.1604 & 0.1562 & 0.1527 & 0.1505 & 0.1485 & 0.1493 \\
Group 4 & 0.4728 & 0.1413 & 0.0785 & 0.0568 & 0.0455 & 0.0407 & 0.0369 & 0.0351 & 0.0345 \\
\hline
\end{tabular}

TABLE 2: RCmvMFE of three-channel synthetic dataset with different $\beta$.

\begin{tabular}{lccccccrrr}
\hline$\beta$ & 1 & 5 & 10 & 15 & 20 & 25 & 30 & 35 & 40 \\
\hline Group 1 & 0.4718 & 0.3318 & 0.3079 & 0.2946 & 0.2847 & 0.2775 & 0.2706 & 0.2632 & 0.2561 \\
Group 2 & 0.4254 & 0.2856 & 0.2609 & 0.2481 & 0.2383 & 0.2314 & 0.2261 & 0.2222 & 0.2192 \\
Group 3 & 0.4409 & 0.2071 & 0.1686 & 0.1559 & 0.1449 & 0.1466 & 0.1439 & 0.1424 & 0.1401 \\
Group 4 & 0.4718 & 0.1406 & 0.0778 & 0.0561 & 0.0455 & 0.0399 & 0.0366 & 0.0348 & 0.0339 \\
\hline
\end{tabular}

TABle 3: Standard deviation of mvMFE with different $\beta$.

\begin{tabular}{lccccccrrr}
\hline$\beta$ & 1 & 5 & 10 & 15 & 20 & 25 & 30 & 35 & 40 \\
\hline Group 1 & 0.0168 & 0.0254 & 0.0293 & 0.0309 & 0.0331 & 0.0350 & 0.0375 & 0.0386 & 0.0418 \\
Group 2 & 0.0192 & 0.0212 & 0.0286 & 0.0354 & 0.0400 & 0.0426 & 0.0402 & 0.0438 & 0.0430 \\
Group 3 & 0.0085 & 0.0101 & 0.0120 & 0.0137 & 0.0166 & 0.0193 & 0.0193 & 0.0200 & 0.0239 \\
Group 4 & 0.0061 & 0.0063 & 0.0048 & 0.0054 & 0.0034 & 0.0046 & 0.0039 & 0.0037 & 0.0039 \\
\hline
\end{tabular}

TABLE 4: Standard deviation of RCmvMFE with different $\beta$.

\begin{tabular}{lccccccrrr}
\hline$\beta$ & 1 & 5 & 10 & 15 & 20 & 25 & 30 & 35 & 40 \\
\hline Group 1 & 0.0206 & 0.0240 & 0.0267 & 0.0275 & 0.0279 & 0.0304 & 0.0355 & 0.0377 & 0.0366 \\
Group 2 & 0.0168 & 0.0216 & 0.0263 & 0.0325 & 0.0350 & 0.0370 & 0.0375 & 0.0381 & 0.0386 \\
Group 3 & 0.0060 & 0.0075 & 0.0105 & 0.0128 & 0.0155 & 0.0174 & 0.0187 & 0.0198 & 0.0207 \\
Group 4 & 0.0063 & 0.0056 & 0.0047 & 0.0040 & 0.0029 & 0.0028 & 0.0030 & 0.0029 & 0.0011 \\
\hline
\end{tabular}

TABLE 5: RCmvMFFE of three-channel synthetic dataset with different $\alpha$.

\begin{tabular}{lcccccccrrr}
\hline$\alpha$ & -0.4 & -0.3 & -0.2 & -0.1 & 0 & 0.1 & 0.2 & 0.3 & 0.4 & 0.5 \\
\hline Group 1 & 0.5850 & 0.7149 & 0.8411 & 0.9505 & 1.0241 & 1.0330 & 0.9312 & 0.6402 & -0.0129 & -1.2583 \\
Group 2 & 0.5626 & 0.6840 & 0.8000 & 0.8977 & 0.9586 & 0.9548 & 0.8422 & 0.5462 & -0.0730 & -1.3068 \\
Group 3 & 0.5156 & 0.6210 & 0.7184 & 0.7954 & 0.8342 & 0.8089 & 0.6791 & 0.3769 & -0.2243 & -1.3868 \\
Group 4 & 0.4463 & 0.5320 & 0.6073 & 0.6605 & 0.6751 & 0.6274 & 0.4815 & 0.1773 & -0.3962 & -1.4684 \\
\hline
\end{tabular}

TABle 6: RCmvMFFE of three-channel synthetic dataset with different $\beta$.

\begin{tabular}{lccccccrrr}
\hline$\beta$ & 1 & 5 & 10 & 15 & 20 & 25 & 30 & 35 & 40 \\
\hline Group 1 & 0.9890 & 0.9194 & 0.8950 & 0.8797 & 0.8676 & 0.8592 & 0.8514 & 0.8446 & 0.8388 \\
Group 2 & 1.0247 & 0.8635 & 0.8339 & 0.8183 & 0.8083 & 0.8020 & 0.7979 & 0.7943 & 0.7907 \\
Group 3 & 1.0418 & 0.7793 & 0.7379 & 0.7248 & 0.7179 & 0.7138 & 0.7117 & 0.7094 & 0.7076 \\
Group 4 & 1.0820 & 0.7091 & 0.6401 & 0.6161 & 0.6049 & 0.5987 & 0.5953 & 0.5933 & 0.5923 \\
\hline
\end{tabular}

possesses more structural complexity than those with less $1 / f$ noise, which is in keeping with the anticipation [8]. Moreover, compared with mvMFE, the standard deviations of RCmvMFE in each scale factor are less, which shows that RCmvMFE combined with refined and composite technique can produce more stable results.

The corresponding results are also depicted in Figure 1; the plots show arithmetic means of 20 independent simulations, and error bars show the standard deviation. Figures 1(a) and 1(b) illustrate the results of mvMFE and
RCmvMFE for three-channel simulation dataset containing independent WGN and $1 / f$ series, respectively. The results are in line with the fact that WGN has less complex structure than $1 / f$ noise and multivariate data with more WGN have less complex structure than those with more $1 / f$ series. For all multichannel data, complexity curves descend as scale factor $\beta$ increases, and the fluctuations of the RCmvMFE curves are significantly smaller than those of the mvMFE. On the other hand, mvMFE and RCmvMFE cannot discriminate these series significantly at large scales, for group 1 and group 2 in 

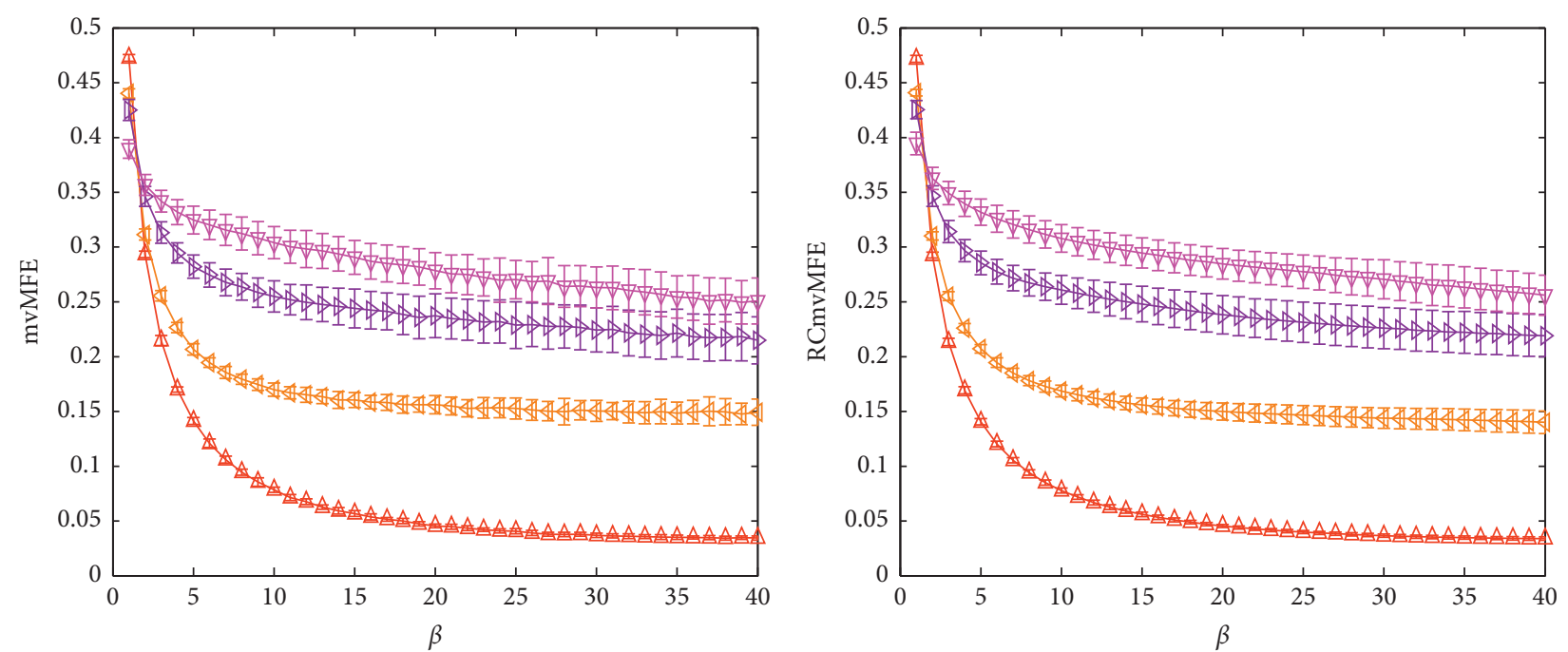

\begin{abstract}
$\triangle$ All three channels contain WGN
$\triangleleft$ Two channles contain WGN, one 1/f noise

$\rightarrow$ One channle contains WGN, others $1 /$ f noise

$\nabla$ All three channels contain $1 / \mathrm{f}$ noise
\end{abstract}

(a) $\triangle$ All three channels contain WGN

$\neg$ Two channels contain WGN, one $1 /$ f noise

$\rightarrow$ One channel contains WGN, others $1 /$ f noise

$\nabla$ All three channels contain $1 /$ f noise

(b)

FIgURE 1: Complexity analyses for three-channel data containing independent WGN and $1 / f$ noise. The plots represent arithmetic means of 20 independent simulations and error bars the standard deviation (SD): (a) mvMFE; (b) RCmvMFE.

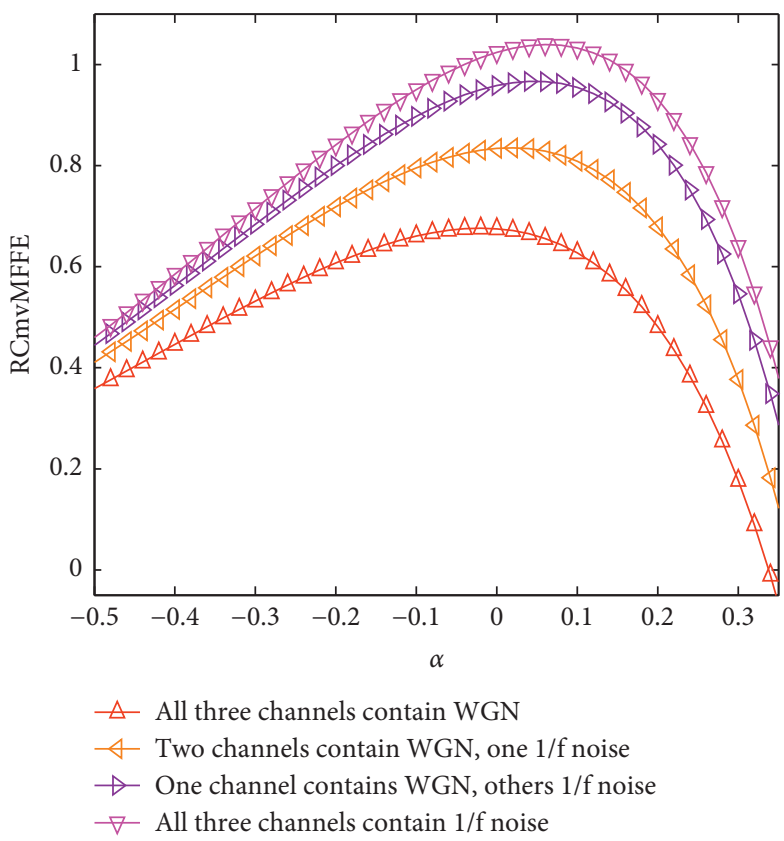

(a)

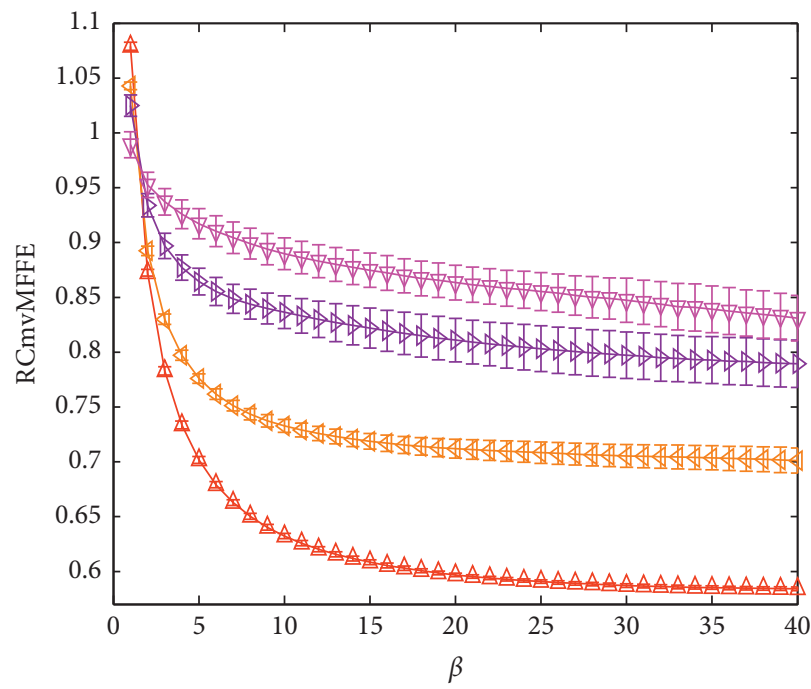

$\triangle$ All three channels contain WGN
$\varangle$ Two channels contain WGN, one $1 /$ f noise
$\triangleright$ One channel contains WGN, others $1 / \mathrm{f}$ noise
$\nabla$ All three channels contain $1 / \mathrm{f}$ noise

(b)

FIGURE 2: Complexity analyses for three-channel data containing independent WGN and $1 / f$ noise. The plots represent an average of 20 independent simulations and error bars the standard deviation (SD): (a) RCmvMFFE vs. $\alpha$ for fixed scale $=20$; (b) RCmvMFFE vs. scale for fixed $\alpha=0.08$.

particular, which may seriously hinder the practical applications. Moreover, it can be found that group 1 and group 2 nearly cannot be separated in Figure 1(a) at large scales, and they can be separated in Figure 1(b).
Then, the RCmvMFFE is chosen to explore the dynamical complexity of the above three-channel synthetic series. Table 5 lists the RCmvMFFE values with different fractional exponent $\alpha$ from -0.4 to 0.5 with step size 0.1 and 
fixed $\beta=20$. According to Table 5 , as $\alpha$ increases, the RCmvMFFE touches the maximum slowly, with $\alpha$ at approximately 0.10 , and then decays quickly. Figure 2 (a) depicts the corresponding RCmvMFFE curves, where all curves are significantly separated, around 0.1 in particular. This further validates the feasibility of the RCmvMFFE measure, which can better distinguish different multichannel synthetic data. Next, Table 6 lists the RCmvMFFE with different time scales $\beta=1,5,10,15,20,25,30,35,40$ and fixed $\alpha=0.08$. Figure 2(b) depicts the corresponding RCmvMFFE curves and shows a similar pattern to that in Figure 1(b), but there is a more significant discrimination. Furthermore, RCmvMFFE method gets larger separation between entropy values in synthetic data than mvMFE and RCmvMFE; hence, RCmvMFFE can distinguish these data more significantly than others. In brief, RCmvMFFE method can conquer the shortcomings of mvMFE and RCmvMFE methods to some extent, and with a suitable fractional exponent $\alpha$ it is relatively stable and can sensitively dig up the inherent properties of different time series.

\section{Complexity Analyses for Multichannel Financial Time Series}

In this section, the complexity dynamics of the multichannel financial time series are explored. We choose 9 important international stock indices from three different regions (i.e., Americas, Europe, and Asia and Pacific) in order to better confirm the application of the proposed complexity measure in real markets. In addition, there are five developed $(D)$ and four emerging $(E)$ stock indices. The 9 indices are BVSP (Brazil, E), MXX (Mexico, E), and GSPC (USA, D) (from Americas market); FCHI (France, $D$ ), GDAXI (Germany, D), and N100 (France, D) (from Europe market); and SSEC (China, E), TWII (China, E), and N225 (Japan, D) (from Asia and Pacific market), respectively. The corresponding data are collected from the Yahoo Financial website (https://in.finance.yahoo. com). The time interval of stock indices (closing price and trading volume) is from Jan. 2, 2003, to Oct. 18, 2019, with approximate 4100 trading days (but there are some slight differences because of the slight different festival and holiday days in the above stock markets, and some individual missing transaction data are supplemented by interpolation method for the sake of synchronicity). In the following work, we adopt logarithmic return given by $r_{t}=\ln p_{t}-\ln p_{t-1}$, where $p_{t}$ represents the closing price of $n$-th trading day. In terms of trading volume, we also adopt the similar logarithmic difference to decline the diversity between them. We also standardize the above data considered to keep uniformity.

4.1. Complexity Measure of Bivariate Stock Indices. In order to explore the different complexity behavior of different financial indices under investigation, we consider a bivariate dataset, whose one channel contains the logarithmic return and the other contains logarithmic trading volume difference of financial indices.
The mvMFE, RCmvMFE, and RCmvMFFE analyses are used to explore the above bivariate dataset. The hyperparameters $n$ and $r$ are fixed as 2 and 0.15 in this paper for simplification purposes, and the corresponding results are exhibited in Figure 3 and Tables 7-9. Figure 3 displays entropy of the bivariate dataset with mvMFE, RCmvMFE, and RCmvMFFE measures with scale $\beta$ from 1 to 30 with step size 1 , and fractional order exponent $\alpha$ is fixed to be 0.10 in RCmvMFFE method. In Figure 3, for all bivariate stock indices, similar to MFE curves, RCmvMFE and RCmvMFFE curves decrease when scale $\beta$ increases. Moreover, entropy curves of FCHI and S\&P500 are under those of SSEC, TWII, and MXX in large scales in RCmvMFE and RCmvMFFE method, which may be because security markets in developed countries are more mature and efficient than in developing countries and possess more random dynamics, while there are long-range dependence in developing countries (similar to $1 / f$ noise) in some sense. This validates that the entropy value of series with long-range correlations is theoretically larger than that of a random signal in large scales [8]. In addition, for mvMFE and RCmvMFE methods, the entropy curves of Asian market are well discriminated, while difference of developed countries is not significant. Although the graphs in Figure 3 could distinguish different stock indices, the gaps between them are relatively small. Furthermore, in the RCmvMFFE analysis, the gap between the two kinds of markets is more significant intuitively, and the gaps between different curves are larger than in mvMFE and RCmvMFE. This means that RCmvMFFE is superior to mvMFE and RCmvMFE in describing the complexity behavior of multichannel financial time series in some sense.

4.2. Complexity Measure of Trivariate Stock Indices in Different Regions. In order to explore the different dynamical complexity behavior of stock indices in different regions under investigation, we consider a trivariate dataset, whose channels contain the logarithmic return of three stock indices from the same region: Americas (BVSP, GSPC, MXX), Europe (FCHI, GDAXI, N100), and Asia and Pacific (SSEC, TWII, N225).

Figures 4(a) and 4(b) show mvMFE and RCmvMFE of the above three-channel dataset. Figures 4(c) and 4(d) show RCmvMFFE for fixed $\alpha=0.08$ and for fixed scale $=20$, respectively. It is interesting that entropy curves of Americas are interwoven with those of Asia and Pacific, and those curves gradually decline and approximate to a fixed number with the increasing scale, but entropy curves of Europe decrease quickly to the minimum value and then gradually increase, and they are always below other curves. Compared with Figure 4(a), Figure 4(b) looks smoother, which confirms that the RCmvMFE is more stable than mvMFE to some extent. Compared with Figure 4(b), Figure 4(c) shows larger gaps, which indicates that the proposed RCmvMFFE has better performance than RCmvMFE in some sense in complexity analysis of short and noisy multichannel financial data.

Tables 10 and 11 list mvMFE and RCmvMFE values of trivariate stock indices with different scale $\beta$, where scale $\beta$ is chosen to be $1,5,10,15,20,25,30,35,40$, 


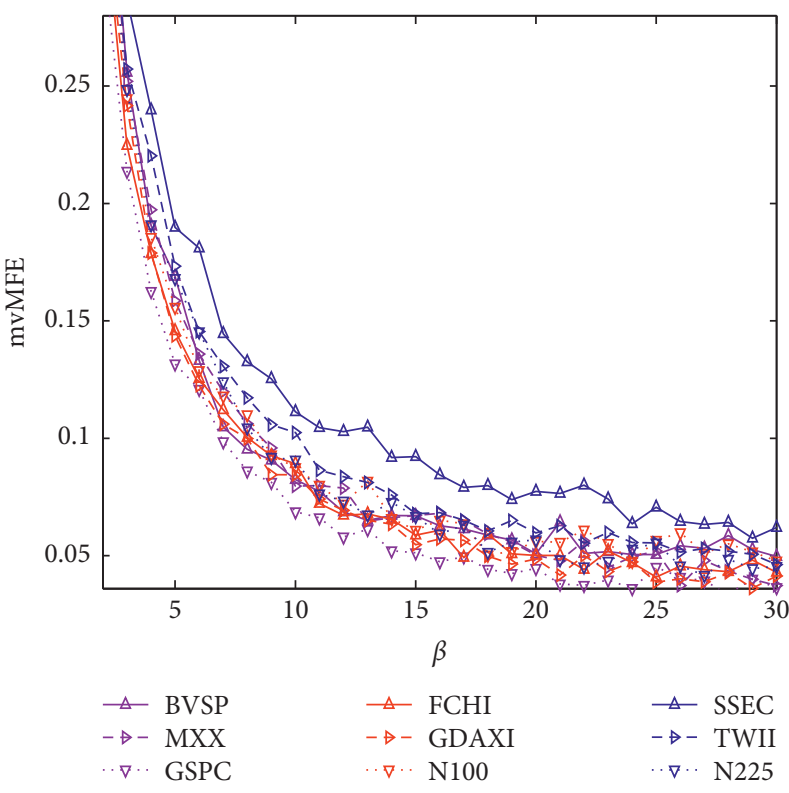

(a)

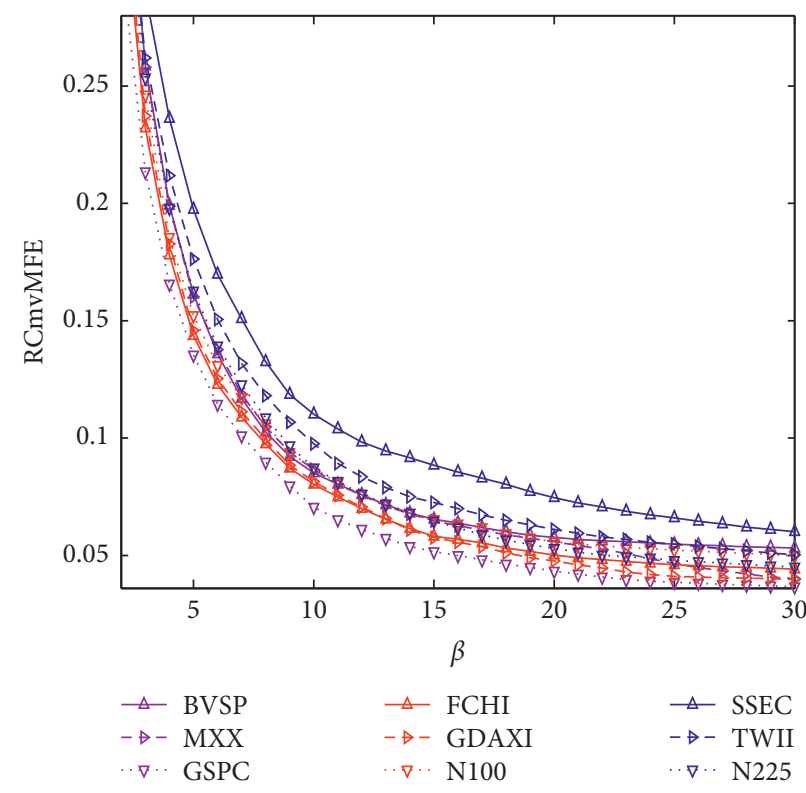

(b)

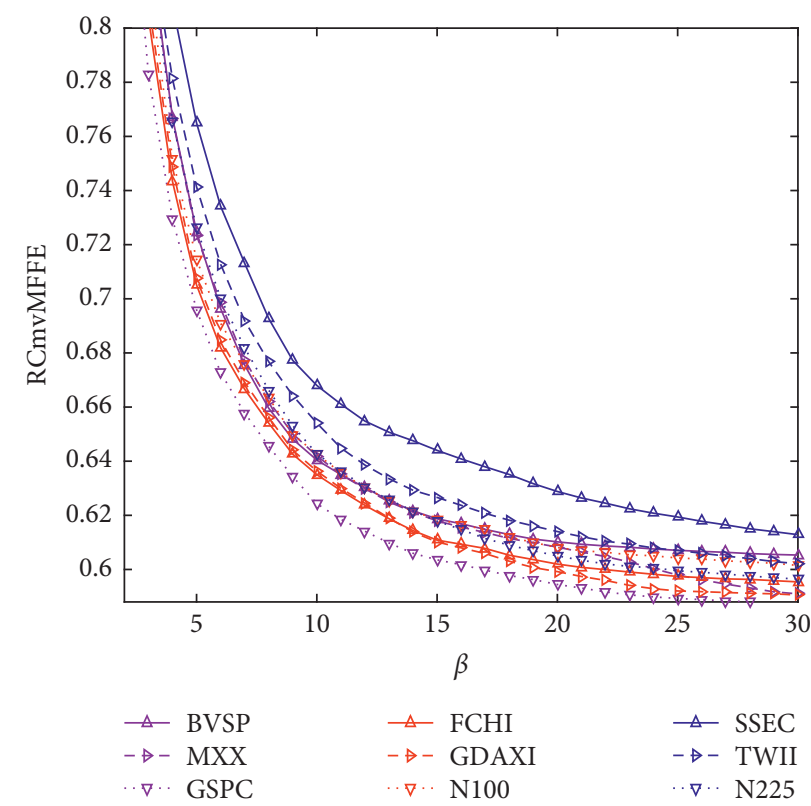

(c)

FIgure 3: Complexity of bivariate stock indices: (a) mvMFE; (b) RCmvMFE; (c) RCmvMFFE.

respectively. For all trivariate stock indices, entropy measures decrease when scale increases, and for a fixed scale $\beta$, entropy measures of SSEC, TWII, and MXX are larger than those of FCHI and S\&P500, which is similar to Figure 3.

Then, the RCmvMFFE analysis is used to explore the dynamical complexity of the trivariate stock indices. Table 12 displays RCmvMFFE values of trivariate stock indices with different time scale factor $\beta$ and fixed $\alpha=0.08$. Table 13 exhibits the RCmvMFFE with different $\alpha$ from -0.4 to 0.4 with step size 0.1 and scale $=20$. As $\alpha$ increases, the RCmvMFFE values touch the maximum and then decay quickly. It can be found that, in Table 13 and Figure 4(d), RCmvMFFE touches the maximum value with $\alpha$ at approximately 0.08 . Figure 4(d) depicts RCmvMFFE curves of return series with different $\alpha$, where all curves are markedly separated. This further validates the effectiveness of the RCmvMFFE measure in discriminating different multichannel financial data. 
TABLE 7: mvMFE of different stock indices with different $\beta$.

\begin{tabular}{lcccccccrc}
\hline$\beta$ & N225 & TWII & SSE & N100 & GDAXI & FCHI & GSPC & MXX & BVSP \\
\hline 1 & 0.6132 & 0.6002 & 0.6150 & 0.5906 & 0.6086 & 0.5879 & 0.5146 & 0.5848 & 0.6039 \\
5 & 0.1681 & 0.1733 & 0.1898 & 0.1559 & 0.1437 & 0.1458 & 0.1317 & 0.1586 & 0.1690 \\
10 & 0.0908 & 0.1024 & 0.1113 & 0.0875 & 0.0845 & 0.0892 & 0.0687 & 0.0797 & 0.0824 \\
15 & 0.0667 & 0.0679 & 0.0922 & 0.0608 & 0.0546 & 0.0587 & 0.0513 & 0.0671 & 0.0670 \\
20 & 0.0568 & 0.0597 & 0.0774 & 0.0561 & 0.0486 & 0.0501 & 0.0446 & 0.0502 & 0.0508 \\
25 & 0.0554 & 0.0554 & 0.0706 & 0.0566 & 0.0387 & 0.0408 & 0.0450 & 0.0520 & 0.0505 \\
30 & 0.0455 & 0.0462 & 0.0619 & 0.0477 & 0.0413 & 0.0429 & 0.0364 & 0.0373 & 0.0497 \\
35 & 0.0442 & 0.0446 & 0.0589 & 0.0486 & 0.0345 & 0.0398 & 0.0388 & 0.0386 & 0.0544 \\
40 & 0.0455 & 0.0429 & 0.0518 & 0.0476 & 0.0309 & 0.0439 & 0.0313 & 0.0360 & 0.0506 \\
\hline
\end{tabular}

TABle 8: RCmvMFE of different stock indices with different $\beta$.

\begin{tabular}{lccccccccc}
\hline$\beta$ & N225 & TWII & SSE & N100 & GDAXI & FCHI & GSPC & MXX & BVSP \\
\hline 1 & 0.6132 & 0.6002 & 0.6150 & 0.5906 & 0.6086 & 0.5879 & 0.5146 & 0.5848 & 0.6039 \\
5 & 0.1627 & 0.1763 & 0.1974 & 0.1522 & 0.1459 & 0.1437 & 0.1354 & 0.1602 & 0.1613 \\
10 & 0.0872 & 0.0976 & 0.1102 & 0.0872 & 0.0815 & 0.0803 & 0.0705 & 0.0866 & 0.0852 \\
15 & 0.0646 & 0.0750 & 0.0884 & 0.0653 & 0.0576 & 0.0582 & 0.0516 & 0.0647 & 0.0654 \\
20 & 0.0529 & 0.0611 & 0.0746 & 0.0557 & 0.0476 & 0.0501 & 0.0433 & 0.0559 & 0.0577 \\
25 & 0.0479 & 0.0548 & 0.0660 & 0.0522 & 0.0411 & 0.0460 & 0.0385 & 0.0465 & 0.0548 \\
30 & 0.0451 & 0.0503 & 0.0601 & 0.0497 & 0.0397 & 0.0441 & 0.0366 & 0.0401 & 0.0532 \\
35 & 0.0436 & 0.0474 & 0.0561 & 0.0474 & 0.0355 & 0.0429 & 0.0349 & 0.0383 & 0.0528 \\
40 & 0.0434 & 0.0473 & 0.0548 & 0.0477 & 0.0358 & 0.0431 & 0.0344 & 0.0381 & 0.0531 \\
\hline
\end{tabular}

TABle 9: RCmvMFFE of different stock indices with different $\beta$.

\begin{tabular}{lccccccccc}
\hline$\beta$ & N225 & TWII & SSE & N100 & GDAXI & FCHI & GSPC & MXX & BVSP \\
\hline 1 & 1.2459 & 1.2304 & 1.2480 & 1.2190 & 1.2404 & 1.2158 & 1.1291 & 1.2121 & 1.2348 \\
5 & 0.7262 & 0.7414 & 0.7650 & 0.7146 & 0.7075 & 0.7051 & 0.6959 & 0.7235 & 0.7247 \\
10 & 0.6427 & 0.6541 & 0.6680 & 0.6426 & 0.6364 & 0.6350 & 0.6243 & 0.6420 & 0.6404 \\
15 & 0.6179 & 0.6266 & 0.6440 & 0.6186 & 0.6102 & 0.6108 & 0.6035 & 0.6179 & 0.6187 \\
20 & 0.6050 & 0.6140 & 0.6288 & 0.6081 & 0.5992 & 0.6020 & 0.5944 & 0.6083 & 0.6102 \\
25 & 0.5995 & 0.6070 & 0.6194 & 0.6042 & 0.5921 & 0.5974 & 0.5893 & 0.5979 & 0.6071 \\
30 & 0.5964 & 0.6021 & 0.6129 & 0.6015 & 0.5905 & 0.5954 & 0.5872 & 0.5910 & 0.6053 \\
35 & 0.5948 & 0.5990 & 0.6085 & 0.5990 & 0.5860 & 0.5941 & 0.5853 & 0.5891 & 0.6049 \\
40 & 0.5946 & 0.5988 & 0.6071 & 0.5993 & 0.5864 & 0.5943 & 0.5847 & 0.5889 & 0.6052 \\
\hline
\end{tabular}

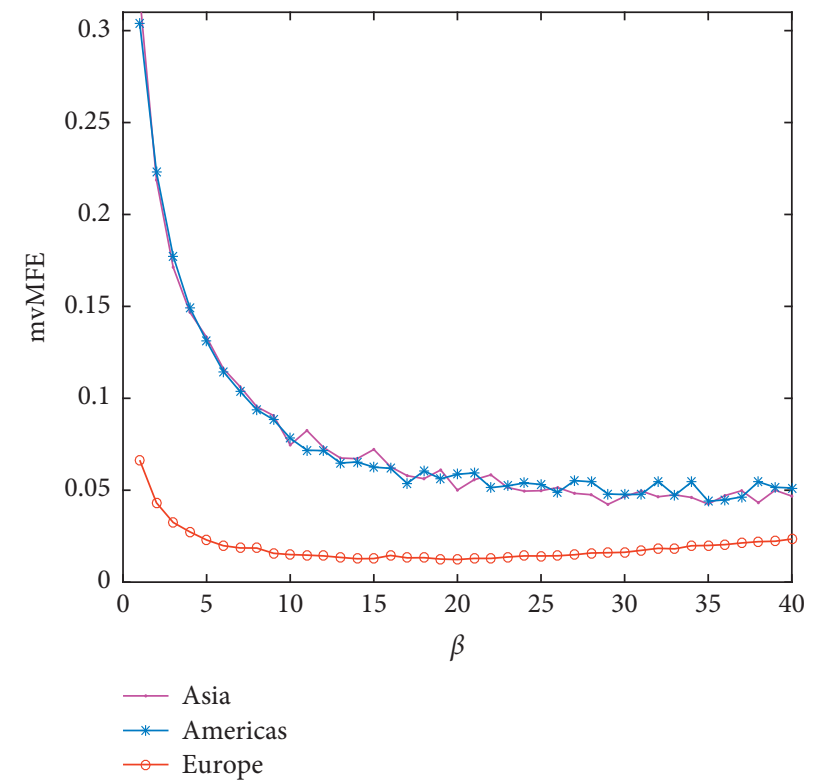

(a)

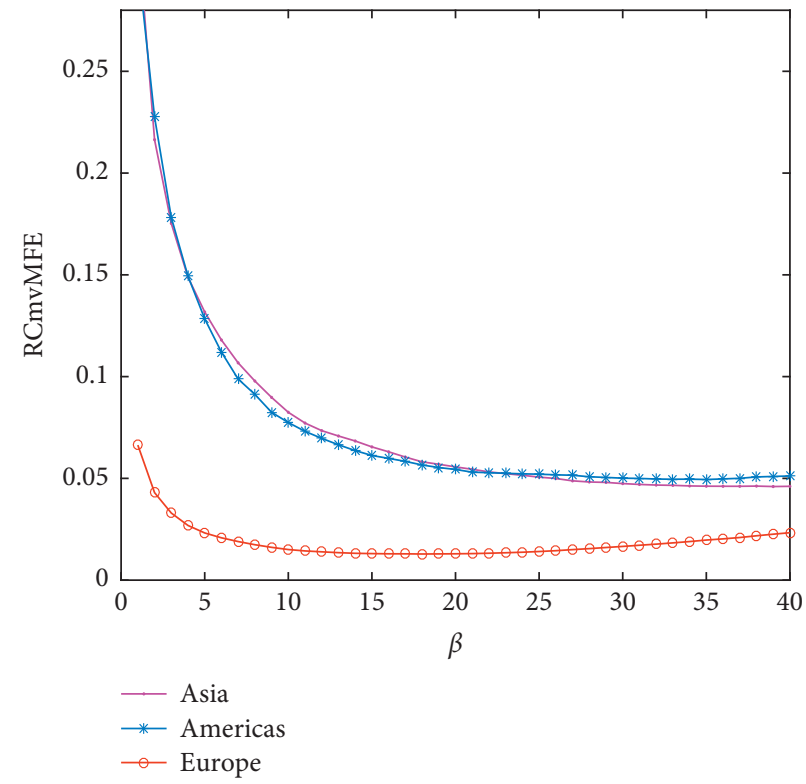

(b)

Figure 4: Continued. 


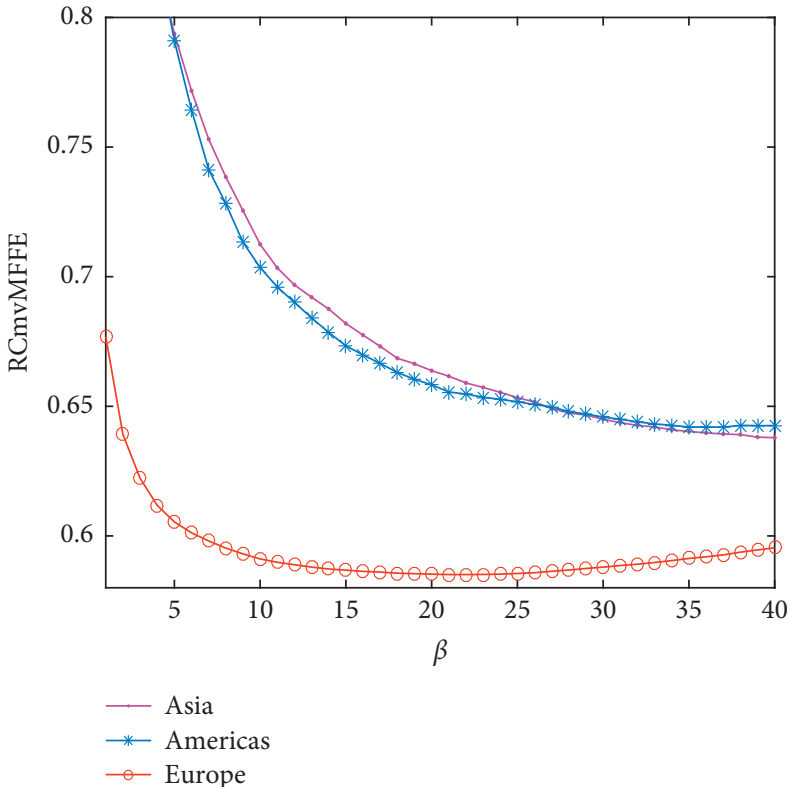

(c)

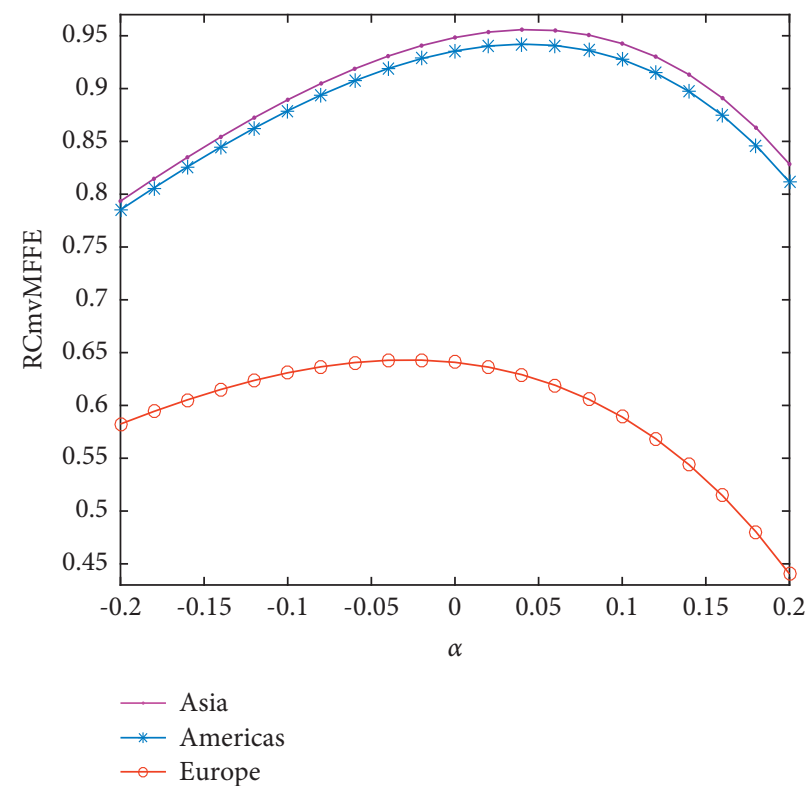

(d)

FIGURE 4: Complexity analyses for three-channel stock indices in different regions: (a) mvMFE; (b) RCmvMFE; (c) RCmvMFFE vs. scale for fixed $\alpha=0.08$; (d) RCmvMFFE vs. $\beta$ for fixed scale $=20$. At a fixed scale of around 25, the entropy values of the Asia and Pacific stock market index and the American stock market index are the same. This may be because they include two emerging market indices. Therefore, we can also see from figure 4 that the stock market index of Asia and Pacific has a downward trend.

TABLE 10: mvMFE of trivariate stock indices with different $\beta$.

\begin{tabular}{lcccccccrr}
\hline$\beta$ & 1 & 5 & 10 & 15 & 20 & 25 & 30 & 35 & 40 \\
\hline Europe & 0.0664 & 0.0229 & 0.0150 & 0.0128 & 0.0123 & 0.0142 & 0.0162 & 0.0199 & 0.0235 \\
Americas & 0.3041 & 0.1311 & 0.0783 & 0.0626 & 0.0587 & 0.0532 & 0.0476 & 0.0440 & 0.0512 \\
Asia & 0.3191 & 0.1332 & 0.0746 & 0.0722 & 0.0501 & 0.0557 & 0.0466 & 0.0425 & 0.0468 \\
\hline
\end{tabular}

TABLE 11: RCmvMFE of trivariate stock indices with different $\beta$.

\begin{tabular}{lccccccccc}
\hline$\beta$ & 1 & 5 & 10 & 15 & 20 & 25 & 30 & 35 & 40 \\
\hline Europe & 0.0664 & 0.0232 & 0.0150 & 0.0130 & 0.0129 & 0.0141 & 0.0166 & 0.0197 & 0.0233 \\
Americas & 0.3041 & 0.1284 & 0.0774 & 0.0612 & 0.0544 & 0.0521 & 0.0501 & 0.0494 & 0.0512 \\
Asia & 0.3191 & 0.1317 & 0.0825 & 0.0655 & 0.0556 & 0.0506 & 0.0474 & 0.0462 & 0.0461 \\
\hline
\end{tabular}

TABLE 12: RCmvMFFE of trivariate stock indices with different $\beta$.

\begin{tabular}{lccccccccc}
\hline$\beta$ & 1 & 5 & 10 & 15 & 20 & 25 & 30 & 35 & 40 \\
\hline Europe & 0.6197 & 0.5725 & 0.5637 & 0.5615 & 0.5614 & 0.5626 & 0.5653 & 0.5688 & 0.5727 \\
Americas & 0.8855 & 0.6881 & 0.6319 & 0.6141 & 0.6066 & 0.6041 & 0.6019 & 0.6012 & 0.6031 \\
Asia & 0.9026 & 0.6919 & 0.6374 & 0.6188 & 0.6080 & 0.6025 & 0.5990 & 0.5976 & 0.5976 \\
\hline
\end{tabular}

TABLE 13: RCmvMFFE of three-channel stock indices in different regions with different $\alpha$.

\begin{tabular}{lcccccccrr}
\hline$\alpha$ & -0.4 & -0.3 & -0.2 & -0.1 & 0 & 0.1 & 0.2 & 0.3 & 0.4 \\
\hline Europe & 0.4145 & 0.4923 & 0.5592 & 0.6035 & 0.6093 & 0.5541 & 0.4033 & 0.1001 & -0.4607 \\
Americas & 0.4648 & 0.5553 & 0.6359 & 0.6948 & 0.7150 & 0.6725 & 0.5300 & 0.2257 & -0.3552 \\
Asia & 0.4685 & 0.5601 & 0.4619 & 0.7020 & 0.7234 & 0.6820 & 0.5403 & 0.2360 & -0.3464 \\
\hline
\end{tabular}




\section{Conclusions}

In this paper, a novel complexity measure for multichannel time series, i.e., RCmvMFFE, is proposed by combining traditional mvMFE measure with fractal theory, refined and composite technique, which can stably and sensitively dig up underlying fractional order behavior and explore structural dynamical complexity in a multivariable complex system. To better comprehend the RCmvMFFE method, the dynamical complexity analyses of multichannel synthetic dataset are comparatively performed with mvMFE, RCmvMFE, and RCmvMFFE. Then, RCmvMFFE method is also employed to explore multiple multichannel financial index series. The experimental analyses report that RCmvMFFE measure is able to deeply and sensitively dig up the market information hidden in the multichannel financial data and can better discriminate different area markets compared to the traditional measures to some extent.

\section{Data Availability}

The data used to support the findings of this study are available from the corresponding author upon request.

\section{Conflicts of Interest}

The authors declare that they have no conflicts of interest.

\section{Acknowledgments}

The authors were supported by the Natural Science Foundation of Universities of Anhui (KJ2019A0141), the Startup Foundation for Introducing Talent of AHPU (2018YQQ024), the Pre-research Project of National Natural Science Foundation of AHPU (2019yyzr15), and the Youth Fund of AHPU (2016YQ29).

\section{References}

[1] L. Calvet and A. Fisher, Multifractal Volatility: Theory, Forecasting, and Pricing, Academic Press, New York, NY, USA, 2008.

[2] W.-C. Chen, "Nonlinear dynamics and chaos in a fractionalorder financial system," Chaos, Solitons \& Fractals, vol. 36, no. 5, pp. 1305-1314, 2008.

[3] J. A. T. Machado, "Complex dynamics of financial indices," Nonlinear Dynamics, vol. 74, no. 1, pp. 287-296, 2013.

[4] R. Cont, "Empirical properties of asset returns: stylized facts and statistical issues," Quantitative Finance, vol. 1, no. 2, pp. 223-236, 2001.

[5] D. Stanislaw, K. Rafal, O. Pawel, R. Rafal, and G. Robert, "Dynamical variety of shapes in financial multifractality," Complexity, vol. 2018, Article ID 7015721, 13 pages, 2018.

[6] W. Zhang and J. Wang, "Nonlinear stochastic interacting dynamics and complexity of financial gasket fractal-like lattice percolation," Physics Letters A, vol. 382, no. 18, pp. 1218-1225, 2018.

[7] R. N. Mantegna and H. E. Stanley, An Introduction to Econophysics: Correlations and Complexity in Finance, Cambridge University Press, Cambridge, Cambridgeshire, UK, 1999.
[8] M. Costa, A. L. Goldberger, and C. K. Peng, "Multiscale entropy analysis of complex physiologic time series," Physical Review Letters, vol. 89, no. 6, pp. 705-708, 2007.

[9] W. Zhang and J. Wang, "Nonlinear stochastic exclusion financial dynamics modeling and time-dependent intrinsic detrended cross-correlation," Physica A: Statistical Mechanics and Its Applications, vol. 482, pp. 29-41, 2017.

[10] T. C. Mills, The Econometric Modeling of Financial Time Series, Cambridge University Press, Cambridge, Cambridgeshire, UK, 1999.

[11] S. M. Pincus, "Approximate entropy as a measure of system complexity," Proceedings of the National Academy of Sciences, vol. 88, no. 6, pp. 2297-2301, 1991.

[12] J. S. Richman and J. R. Moorman, "Physiological time-series analysis using approximate entropy and sample entropy," American Journal of Physiology. Heart and Circulatory Physiology, vol. 278, no. 6, pp. H2039-H2049, 2000.

[13] W. Chen, J. Zhuang, W. Yu, and Z. Wang, "Measuring complexity using FuzzyEn, ApEn, and SampEn," Medical Engineering \& Physics, vol. 31, no. 1, pp. 61-68, 2009.

[14] H. Niu, J. Wang, and C. Liu, "Analysis of crude oil markets with improved multiscale weighted permutation entropy," Physica A: Statistical Mechanics and Its Applications, vol. 494, pp. 389-402, 2018.

[15] C. Bandt and B. Pompe, "Permutation entropy: a natural complexity measure for time series," Physical Review Letters, vol. 88, Article ID 174102, 2002.

[16] A. K. Vidybida, "Calculating permutation entropy without permutations," Complexity, vol. 2020, Article ID 7163254, 9 pages, 2020.

[17] W. Chen, Z. Wang, H. Xie, and W. Yu, "Characterization of surface EMG signal based on fuzzy entropy," IEEE Transactions on Neural Systems and Rehabilitation Engineering, vol. 15, no. 2, pp. 266-272, 2007.

[18] Y. Wang, S. Zheng, W. Zhang, G. Wang, and J. Wang, "Fuzzy entropy complexity and multifractal behavior of statistical physics financial dynamics," Physica A: Statistical Mechanics and Its Applications, vol. 506, pp. 486-498, 2018.

[19] Y. Li, B. Miao, W. Zhang, P. Chen, J. Liu, and X. Jiang, "Refined composite multiscale fuzzy entropy: localized defect detection of rolling element bearing," Journal of Mechanical Science and Technology, vol. 33, no. 1, pp. 109-120, 2019.

[20] H. H. Anne, "Multivariate refined composite multiscale entropy analysis," Physics Letters A, vol. 380, pp. 1426-1431, 2016.

[21] S. Banerjee, S. K. Palit, S. Mukherjee, M. R. Ariffin, and L. Rondoni, "Complexity in congestive heart failure: a timefrequency approach," Chaos, vol. 26, Article ID 033105, 2016.

[22] J.-B. Liu, J. Zhao, H. He, and Z. Shao, "Valency-based topological descriptors and structural property of the generalized sierpiński networks," Journal of Statistical Physics, vol. 177, no. 6, pp. 1131-1147, 2019.

[23] D. Valério, J. J. Trujillo, M. Rivero, J. A. T. Machado, and D. Baleanu, "Fractional calculus: a survey of useful formulas," The European Physical Journal - Special Topics, vol. 222, no. 8, pp. 1827-1846, 2013.

[24] J. Machado, "Fractional order generalized information," Entropy, vol. 16, no. 4, pp. 2350-2361, 2014.

[25] J. B. Liu, J. Zhao, J. Min, and D. Cao, "On the Hosoya index of graphs formed by a fractal graph," Fractals, vol. 27, no. 8, Article ID 1950135, 2019.

[26] A. Humeau-Heurtier, C.-W. Wu, and S.-D. Wu, "Refined composite multiscale permutation entropy to overcome 
multiscale permutation entropy length dependence," IEEE Signal Processing Letters, vol. 22, no. 12, pp. 2364-2367, 2015.

[27] M. U. Ahmed and D. P. Mandic, "Multivariate multiscale entropy: a tool for complexity analysis of multichannel data," Physical review. E, Statistical, nonlinear, and soft matter physics, vol. 84, no. 6, Article ID 061918, 2011.

[28] F. Takens, Detecting Strange Attractors in Turbulence, Springer, New York, NY, USA, 1981.

[29] L. Cao, A. Mees, and K. Judd, "Dynamics from multivariate time series," Physica D: Nonlinear Phenomena, vol. 121, no. 12, pp. 75-88, 1998. 